\title{
Lymph transport in rat mesenteric lymphatics experiencing edemagenic stress
}

\author{
Elaheh Rahbar ${ }^{1}$, Tony AkI ${ }^{2}$, Gerard L. Coté ${ }^{2}$, James E. Moore Jr. ${ }^{3}$, and David C. Zawieja ${ }^{4}$ \\ ${ }^{1}$ Center for Translational Injury Research, Department of Surgery, University of Texas Health \\ Science Center, Houston, TX 77030, USA \\ 2Department of Biomedical Engineering, Texas A\&M University, 5045 Emerging Technologies \\ Building, 3120 TAMU, College Station, TX 77843-3120, USA \\ ${ }^{3}$ Department of Bioengineering Imperial College London South Kensington Campus Royal School \\ of Mines Building, Room 4.14 London SW7 2AZ, United Kingdom \\ 4Department of Systems Biology and Translational Medicine, Cardiovascular Research Institute, \\ Division of Lymphatic Biology, College of Medicine, Texas A\&M Health Science Center, 702 SW \\ H.K. Dodgen Loop, Temple, TX 76504, USA
}

\section{Abstract}

Objective-To assess lymphatic flow adaptations to edema, we evaluated lymph transport function in rat mesenteric lymphatics under normal and edemagenic conditions in situ.

Methods-Twelve rats were infused with saline (intravenous infusion, $0.2 \mathrm{ml} / \mathrm{min} / 100 \mathrm{~g}$ body weight) to induce edema. We intravitally measured mesenteric lymphatic diameter and contraction frequency, as well as immune cell velocity and density before, during and after infusion.

Results-A 10-fold increase in lymph velocity $(0.1-1 \mathrm{~mm} / \mathrm{s})$ and a 6-fold increase in flow rate $(0.1-0.6 \mu \mathrm{L} / \mathrm{min})$, were observed post-infusion, respectively. There were also increases in contraction frequency and fractional pump flow 1-minute post-infusion. Time-averaged wall shear stress increased 10 fold post-infusion to nearly 1.5 dynes $/ \mathrm{cm}^{2}$. Similarly, maximum shear stress rose from 5 dynes $/ \mathrm{cm}^{2}$ to 40 dynes $/ \mathrm{cm}^{2}$.

Conclusions-Lymphatic vessels adapted to edemagenic stress by increasing lymph transport. Specifically, the increases in lymphatic contraction frequency, lymph velocity, and shear stress were significant. Lymph pumping increased post-infusion, though changes in lymphatic diameter were not statistically significant. These results indicate that edemagenic conditions stimulate lymph transport via increases in lymphatic contraction frequency, lymph velocity and flow. These changes, consequently, resulted in large increases in wall shear stress, which could then activate NO pathways and modulate lymphatic transport function.

\section{Keywords}

lymph flow; edema; contractility; frequency; shear stress

Corresponding Author: Dr. David C. Zawieja, Department of Systems Biology and Translational Medicine, Texas A\&M Health Science Center, 702 SW H.K. Dodgen Loop, Temple, TX 76504 USA, dzawieja@ medicine.tamhsc.edu, Phone: (254) 231-1500. 


\section{Introduction}

The lymphatic system transports fluid from the interstitium through a series of valves, nodes and pre- and post-nodal lymph vessels that empty into the great veins of the thorax at the juncture of the subclavian and internal jugular veins $[9,35,39,50,51]$. This system is a key component in overall body fluid balance, macromolecular homeostasis, lipid absorption, and immune function. All of these functions rely on the generation and regulation of lymph flow along the lymphatic network. Normally it is thought that the removal of interstitial fluid via lymph formation is balanced with the extravasation of fluids from the exchange blood vessels to maintain a relatively constant interstitial fluid volume [22,25,26,51]. In healthy individuals, there must also be a coupling between the rate of lymph formation in the lymph capillaries and the ability of the downstream lymphatic vessels to transport lymph through the lymphatic network and on into the venous circulation $[4,5,24,50]$. Edema occurs when there is an imbalance in any of these processes, i.e. with a pathological increase in interstitial fluid volume, either with abnormally elevated blood vessel extravasation, with impaired lymphatic function (either anatomically or functionally deranged), or in situations where there is a relative distortion of both factors [34,36,37,40,43,44]. Historically, lymphedema has been an edema that is associated with a primary defect in the lymphatic side of this balance. Lymphedema has particularly been a problem in developing countries where there is a high prevalence of lymphatic filariasis, a parasitic worm that infects lymphatic vessels and impairs lymph flow, with 120 million affected individuals worldwide and 1.2 billion people with the risk of infection globally [8,49]. Indeed, the World Health Organization launched its Global Programme to Eliminate Lymphatic Filariasis (GPELF) in 2000 with the goal to eliminate lymphatic filariasis as a public-health problem by 2020 . However, lymphedema in the United States is most prevalent amongst breast cancer patients; where up to $22 \%$ of women develop lymphedema in their arms within 3 years of nodal dissection surgery [12,34,37,40,42]. Chronically, in addition to tissue fluid accumulation, there is an increase in protein concentration, inflammation, recurrent infection, fibrosis and adipose content which causes pain, swelling and immobility for the individual. Despite widespread recognition of these serious health problems, there is a poor understanding of the mechanisms involved and a lack of effective treatments for lymphedema.

Lymph formation occurs in the interstitium; when water, solutes, and plasma proteins escape from the blood capillaries into the interstitial compartment [22,26,51]. Flow across the capillary wall is a process of plasma ultrafiltration through a semi-permeable membrane. The primary driving force for ultrafiltration is capillary blood pressure $\left(P_{c}\right)$, whereas the oncotic pressure of plasma proteins $\left(\pi_{\mathrm{p}}\right)$ opposes filtration [24-26]. Therefore, an increase in blood pressure would result in an increase in $P_{c}$, thereby increasing the flux of fluid entering the interstitium. Alternatively, a reduction in plasma oncotic pressure would impair reabsorption back into the vascular space and consequently induce edema in the interstitial space. Once fluid accumulates in the interstitium, the initial lymphatic vessels must take it up or lymphedema can develop. While there is some debate on the exact mechanism regulating the formation of lymph into the initial lymphatics, it is generally believed that the average prevailing pressure gradient between the interstitial fluid and initial lymphatic changes periodically to allow lymph to enter the initial lymphatics via the primary valves 
$[22,27,41,45]$. Additionally, viscous fluid stresses and the expansion and compression of neighboring tissues aid in the uptake of lymph into the initial lymphatics. In humans, approximately $4-8$ liters of fluid is transported by the lymphatics per day [32,50,51].

Once interstitial fluid enters the lymphatic system, it needs to be transported "uphill" through the lymphatic network including the nodes towards the return point in the great veins. Studies have shown that the lymphatic system relies on two general types of pumps to transport lymph, namely the intrinsic and extrinsic pumps [15-17,50,52,53]. Collecting lymphangions, in particular, possess smooth muscle cells and many have the ability to contract intrinsically and actively pump lymph forward $[15,17,52,53]$. The extrinsic pump refers to the pumping from contraction/relaxation cycles induced by adjacent structures acting upon the lymphatic (i.e. pulsating arteries, respiratory activities, GI peristalsis and skeletal muscle contractions). We propose that edemagenic conditions will stimulate both lymph pumps to increase lymph transport.

This study seeks to investigate and quantify lymphatic function under normal and edemagenic conditions in situ, using our previously established high speed imaging system to measure lymph velocity and flow $[1,3,5,13,14]$. We conducted these studies in a wellestablished in vivo model of lymph transport in the rat mesenteric lymphatics. Edemagenic stress was induced by the intravenous administration of saline $(0.9 \% \mathrm{NaCl})$ via the femoral vein in rats [5]. This infusion induces a reduction in plasma oncotic pressure and slight increases in blood pressure, thereby increasing the pressure gradient across the capillary wall and resulting in an increase in fluid volume in the interstitium, resulting in an increase in lymph formation [5]. This increased fluid volume will increase lymph formation in the initial lymphatic vessels and thus more fluid will enter the lymphatic network. We hypothesize that lymphatic vessels will accommodate the increased lymph formation by increasing lymph transport downstream by stimulating pump function. This will result in changes in lymph pump contraction frequency, lymph pump stroke volume, lymph velocity and flow rate.

\section{Materials and Methods}

\section{Animals}

Twelve male Sprague Dawley rats weighing 200-300 g were used for this study. All animals were housed in an environmentally controlled vivarium, approved by the American Association for Accreditation of Laboratory Animal Care, and allowed access to a standard pellet diet and water. Rats were fasted 15-20 hours before experimentation with water available ad libitum.

\section{Surgical Preparation}

The rats were anesthetized with intramuscular injections of fentanyl-droperidol $(0.3 \mathrm{~mL} / \mathrm{kg})$ and diazepam $(2.5 \mathrm{mg} / \mathrm{kg})$. Supplemental doses of the anesthetic were provided as necessary. The right femoral vein was cannulated with PE-50 tubing for intravenous fluid (saline) administration. The cannula was connected to a syringe with a 23-gauge adapter. Saline $(0.9 \% \mathrm{NaCl})$ was prepared and sterile filtered for all experiments. To prevent clotting 
of the cannula, $0.05 \mathrm{ml}$ of heparin was diluted in $1 \mathrm{ml}$ of saline and a small volume (0.1-0.5 $\mathrm{ml}$ ) was perfused into the femoral vein. Once the cannula was firmly in place, the rat was transported to the preparation board. To gain access to the mesentery, a midline abdominal incision was made and a loop of small intestine was exteriorized. The mesentery was draped over a glass semicircular viewing pedestal $(12.5 \times 25 \mathrm{~mm})$, and with the aid of a dissecting microscope, a muscular collecting lymphatic was centered on the viewing pedestal. The exposed tissue was constantly suffused with warm albumin physiologic saline solution (in mM: $145.00 \mathrm{NaCl}, 4.7 \mathrm{KCl}, 2.0 \mathrm{CaCl}_{2}, 1.17 \mathrm{MgSO}_{4}, 1.2 \mathrm{NaH}_{2} \mathrm{PO}_{4}, 5.0$ dextrose, 2.0 sodium pyruvate, $0.02 \mathrm{EDTA}, 3.0 \mathrm{MOPS}$, and $10 \mathrm{~g} / \mathrm{L}$ bovine serum albumin). The solution was prewarmed to $37^{\circ} \mathrm{C}$ and had the $\mathrm{pH}$ adjusted to 7.4. This suffusion prevented the lymphatic vessel and mesentery bed from drying up and further kept the animal's core and tissue temperature at $36-38^{\circ} \mathrm{C}$ for the entire duration of the experiment. In addition to monitoring body temperature, a pulse oximeter, attached to the rat's foot, was used to monitor blood oxygen levels and heart rate. The preparation was then transferred to a Zeiss intravital microscope for imaging.

\section{Saline Infusion \& Image Acquisition}

Using an $80 \mathrm{~mm}$ projective lens and 10x water immersion objective we were able to view the lymphatic vessels with a depth of field of approximately $14 \mu \mathrm{m}$. The microscope was connected to a high resolution, high speed CCD camera (Phantom V5.2 1152x896,Vision Research Inc.) which captured images at 500 fps. The camera was triggered to capture a burst of 8 images at $500 \mathrm{fps}$ then wait for $34 \mathrm{~ms}$ to take another burst of images $[1,3,13,14]$. This allowed us to extend the imaging time to capture multiple contraction cycles. To extend the imaging time even further, the camera's field of view was reduced as much as possible to cover only the area occupied by the vessel during all times and to reduce the capture of unnecessary data. Data were recorded in intervals long enough to acquire at least 2 contractile cycles. Only lymphatics that exhibited spontaneous rhythmic contractile patterns were selected for this study.

An initial acquisition was taken before any volume infusion at the beginning of the experiment to serve as a baseline measurement. To make up for insensible water loss that could affect lymph flow through an in situ experiment of this duration, we administered a slow constant perfusion of saline at $0.004 \mathrm{ml} / \mathrm{min} / 100 \mathrm{~g}$ body weight for 10 minutes using a syringe pump. This flow rate has been estimated to be equal to the normal urine flow and respiratory water losses in rats [5]. Sequences of images were taken during this slow infusion (usually 1-2 sets). Once the slow infusion was done we acquired data, which was then subsequently used as a second control period to which all others were compared; this acquisition was taken to ensure flow was normal. To induce hyperdynamic conditions, the infusion rate was increased to $0.2 \mathrm{ml} / \mathrm{min} / 100 \mathrm{~g}$ body weight for 10 minutes. This produces a significant hypotonic and hypooncotic fluid volume, which we have shown in the past to increase lymph pumping within 10 minutes from the start of infusion [5]. Images were captured both during and after the high infusion, up to 25 minutes post fast infusion. In total, 7-12 sets of images were taken from each animal over the span of the entire experiment. Upon completion of the experiment, rats were euthanized with pentobarbital $(120 \mathrm{mg} / \mathrm{kg}$ of body weight IP). 


\section{Image Processing}

Images acquired from the experiments were processed with an in-house program, written in Matlab (version 2009b, MathWorks Inc. Natick, MA), to obtain values for lymphatic vessel diameter, lymphocyte velocity and lymphocyte density. Vessel diameter and lymphocyte velocities were continuously recorded using a correlation-based pattern-tracking algorithm described previously by Akl et al. [1-3]. This algorithm was a modification to the previously published work by Dixon et al. [13,14]. Briefly, each set of 4 consecutive images were used to filter out background noise and create one image with enhanced particles to background contrast. Immune cells, presumably the majority of which are lymphocytes, in the filtered images were tracked to measure their velocity. Edge detection was used to measure vessel diameter, cell density and cell position. Lymphocyte density was calculated to ensure that we did not induce an inflammatory response. Further analysis involved calculating lymph pump contraction frequency, fractional pump flow, lymph flow rate, and wall shear stress from the obtained velocity and diameter measurements, using general methods we used previously [1-3,14].

\section{Statistical Analysis}

Data from the twelve rats were organized into eight time categories; namely, control (time $=0, \mathrm{~N}=12$ ), slow infusion (time $=0-10$ minutes, $\mathrm{N}=11$ ), edemagenic/fast infusion (time $=10-20$ minutes, $\mathrm{N}=12$ ), 1 minute post infusion (time = minute 21 experimental time, $\mathrm{N}=4$ ), 3-5 minutes post edemagenic infusion (time $=23-25$ minutes, $\mathrm{N}=11$ ), 10-12 minutes post infusion (time $=30-32$ minutes experimental time, $\mathrm{N}=11$ ), 13-19 minutes post edemagenic infusion (time $=33-39$ minutes, $\mathrm{N}=6$ ) and greater than 20 minutes post edemagenic infusion (time $>40$ minutes, $\mathrm{N}=4$ ). The shaded boxes and time axes in the figures represent these eight categories. All data are expressed as means \pm standard error (SE). A generalized estimating equation (GEE) model, with compound symmetry covariance structure was used to compare each outcome variable to its respective control set, with a 95\% confidence interval $(a=0.05)$. This type of longitudinal analysis was chosen over repeated measures ANOVA due to its robustness in handling unequal sample sizes over time, while maintaining the unique relationships between each repeated measurement per rat.

\section{Results \\ Vessel Diameter}

Average lymphatic diameter was greatest $13-19$ minutes post infusion $(d=116.7 \mu \mathrm{m})$ but this increase in vessel diameter was not statistically significant. Diastolic diameters $\left(D_{d}\right)$ ranged from 50-178 $\mu \mathrm{m}$ and systolic diameters $\left(\mathrm{D}_{\mathrm{s}}\right)$ ranged from $20-169 \mu \mathrm{m}$ throughout the experiment. When we normalized the data to the corresponding control diastolic diameter for each vessel, there was a slight increase in diastolic diameter and decrease in systolic diameter both during fast infusion and one minute post infusion, but differences remained statistically insignificant (p-value $>0.05$ ) as shown in Figure 1a. There was a modest albeit non-significant increase in the normalized diastolic ( 18\%) and systolic ( 35\%) lymphatic diameters 13-19 minutes after infusion. However, it should be noted that p-value was lowest (p-value $<0.1$ ) during the last time point (i.e. $>20$ minutes post-infusion) for average 
diameter, diastolic diameter and systolic diameter suggesting that there could be a slight increase in vessel diameter at the end of the experiment. The pulse diameter (i.e. difference between diastolic and systolic diameter) began to increase and reached its maximum at one minute post infusion, but this increase was statistically insignificant ( $\mathrm{p}$-value $=0.453$, Figure 1b). It is important to note that the standard deviation at each time point gradually increased as the mean pulse diameter increased meaning that there was a greater variation in the vessel diameter response to edemagenic stress, which may explain why statistical significance was not observed.

\section{Lymphocyte Velocity and Flow Rate}

Average lymphocyte velocity was $0.1 \mathrm{~mm} / \mathrm{s}$ under control conditions and increased steadily upon administration of the fast saline infusion. Average velocity increased up to 10 times and sustained velocities greater than $1 \mathrm{~mm} / \mathrm{s}$ at 20 minutes post-infusion. Average lymph velocities increased throughout the entire infusion periods and were significantly higher at all time points following 3 minutes post-infusion (p-value $<0.05$, Figure 2 ). Similarly, changes in average flow rate were statistically significant at one minute post-infusion, 3-5 minutes post infusion, and 13-19 minutes post-infusion reaching a maximum value of 0.6 $\mu \mathrm{L} / \mathrm{min}$ (p-value $<0.05$, Figure 2).

\section{Contraction Frequency and Fractional Pump Flow}

There was over a $50 \%$ increase in contraction frequency at one-minute post infusion. In fact, contraction frequency significantly increased both during fast infusion and one minute postinfusion (p-value $<0.05$, Figure 3 ). Ejection fraction was determined from diastolic and systolic diameters and used to calculate fractional pump flow, which is defined as the product of ejection fraction and frequency. The data showed a significant increase in fractional pump flow during fast infusion, one-minute post infusion and 10-12 minutes postinfusion (Figure 3).

\section{Wall Shear Stress}

Average wall shear stress for control was 0.12 dynes $/ \mathrm{cm}^{2}$ with a maximum shear stress of 5 dynes $/ \mathrm{cm}^{2}$. As the experiment progressed, average wall shear stress increased by an order of magnitude to nearly 1.5 dynes $/ \mathrm{cm}^{2}$. The increases in average wall shear stress were statistically significant at all time points post-infusion with the exception of the 13-19 minute mark (Figure 4a). Maximum wall shear stress rose to 40 dynes $/ \mathrm{cm}^{2}$ during fast saline infusion; $8 \mathrm{X}$ higher than the control maximum value for shear stress. A larger increase (up to $11 \mathrm{X}$ ) was observed at one minute post-infusion. Due to the large variation in maximum shear stress values amongst animals, we normalized the max shear stress with respect to the control value for max shear stress. There were statistically significant increases in normalized maximum wall shear stress during fast infusion and one minute post-infusion (pvalue $<0.05$, Figure $4 \mathrm{~b}$ ). Wall shear stress remained elevated at the end of the experiment.

\section{Lymphocyte Density}

To ensure that the saline infusion did not elicit an inflammatory response, we quantified cell density. Average cell density observed during our experiments was about 150,000 cells $/ \mathrm{mL}$. 
Our data shows that cell density decreased $15 \%$ from control over the duration of the experiment, which was expected, as the saline infusion would cause some dilution of the lymph. However, this decrease in lymphocyte density was not statistically significant (pvalue $>0.05$, Figure 5). Given that lymphocyte tracking is crucial to our velocity measurements, we captured high-speed video when the cells were observed, not continuously throughout the experiment. It is, therefore, possible that actual time averaged lymphocyte densities may be somewhat different than our reported values.

\section{Discussion}

The most common cause of lymphedema in the western world is the result of surgery and oncologic therapies. With the growing prevalence of breast and pelvic cancer, there has been a subsequent rise in secondary lymphedema cases $[12,36,38,40,42]$. Therefore, characterizing lymphatic function and its response to an edemagenic stress is crucial to our understanding of these pathologies. Our knowledge of the mechanisms regulating the transport of fluid and lymphatic contractility remains fairly rudimentary. In this study we evaluated lymphatic function and for the first time, measured lymph pump activity simultaneously with lymph flow, lymph velocity and lymphatic wall shear stress under normal and edemagenic conditions in the rat mesentery in situ. Our results showed a significant increase in lymph flow rate, lymph velocity, lymphatic wall shear stress, lymphatic contraction frequency and fractional pump flow. These results strongly suggest the mechanosensing nature of lymphatic vessels where lymph pressure and shear stress play important roles in modulating lymphatic contractility, the lymph pump and thus lymph transport.

Healthy lymphatic vessels from the rat mesentery were shown to quickly adapt to the increased fluid volume (i.e. edemagenic stress) induced by the saline infusion. The increase in lymph flow rate, lymphatic contraction frequency and the fractional pump flow clearly indicate increased lymphatic contractile activity. These results closely parallel the work by Benoit et al. who performed a similar experiment but did not measure actual lymph flow rate or velocity [4,5]. It should be noted that Benoit et al. assessed lymphatic response up to 10 minutes from the start of infusion, which corresponds to our "fast infusion" time point. Therefore, Benoit et al. focused on evaluating the immediate response whereas; in this study we evaluated lymphatic function up to 30 minutes post-infusion. The differences between our findings from the immediate and longer-term responses are discussed in more detail later on.

As soon as one-minute post-infusion, lymph flow doubled and flow rate eventually reaching up to six times the control. The change in measured lymph flow is similar in magnitude to the calculated changes in volume flow in our previous study [4,5]. Flow was predominantly unidirectional, although oscillations were observed. We normalized maximum velocity and shear stress to their corresponding average velocity and shear stress to evaluate oscillatory flow and discovered no significant findings due to the wide range of variation. Similarly ejection fraction and frequency of contractions increased resulting in a large change in fractional pump flow. Despite statistically insignificant changes in average vessel diameter, small changes in diastolic and systolic diameters caused the ejection fraction to increase 
during fast infusion and one-minute post infusion thereby affecting fractional pump flow. This variable clearly demonstrated the change in lymphatic contractility to accommodate the increased fluid volume and is similar in direction to what we previously observed $[4,5]$. Notably, changes in lymph pump frequency and fractional pump flow were significant during the fast saline infusion (indicative of the acute pump activation) while changes in lymph velocity and flow rate were increasing throughout the infusion periods and achieves significance after the fast infusion (indicative of the increasing influence of the hypotonic fluid volume on interstitial lymph hydrodynamics). Changes in average shear stress experienced by the lymphatic vessels were quite large reaching up to 10 times the control values at 20 minutes post-infusion. On the other hand, maximum wall shear stress was highest during fast infusion and one-minute post-infusion, again indicative of the increased lymph pump pulse diameter during these time periods.

These results illustrate the immediate changes in frequency, fractional pump flow, and maximum shear stress during the fast infusion. Altogether, these responses suggest that the saline infusion affects lymph formation almost instantly, eliciting an increase in lymph flux at the initial lymphatic vessels. Consequently, the lymphatic vessels begin to contract more frequently to accommodate the increased lymph volume. The increase in frequency and relatively unchanged vessel diameter explain the significantly increased maximum wall shear stress experienced by the vessel at this time point. We believe that this sudden increase in maximum shear stress activates the release of nitric oxide (NO) from the lymphatic endothelium. We have previously shown that flow-induced NO plays important roles in the modulation and regulation of the active lymph pump as well as, increased production of nitric oxide by the lymphatic endothelium under similar conditions $[6,7,15-18,21]$. Thus, we postulate that the release of NO begins during fast infusion when maximum shear stress is greatest, thereby affecting vessel frequency such that normal shear stress values are restored. The results from this study support this idea, as we observe the greatest vessel diameter at the 33-39 minute mark while frequency decreases with time. Vessel pumping increased by $50 \%$ at one-minute post-infusion and gradually decreased with time, presumably as a result from the complex changes in shear and stretch mediated effects. Interestingly, flow rate is greatest at this time point (33-39 minutes) as well, which correlates to our previous work and findings from Quick et al. who illustrated the conduit behavior of lymphatic vessels $[29,47,48]$. Thus, our results illustrate increased pumping as an immediate response to edemagenic stress followed by a "conduit-like" response in the long term to accommodate the increased lymph volume. Assuming we extended the experiment longer, we'd expect to eventually observe normal lymphatic function as the interstitial volume is returned back to normal.

While the findings of this study illustrate both immediate and longer-term responses of lymphatic vessels to edemagenic stress (up to 30 minutes post infusion), there is a need to elucidate specific mechanisms for the observed lymphatic response. The saline infusion via the femoral vein causes an increase in lymph formation starting at the interstitial level thereby eliciting changes in pressure and shear on the initial lymphatics [24-26]. The saline infusion results in greater lymph formation and an increased flux of fluid into the initial lymphatic vessels. Consequently, the collecting lymphangions must adapt to transport this excess lymph fluid. We postulate that increases in pressure and shear stress due to the 
increased fluid volume regulate the immediate increase in lymphatic contractility. As seen by our data, under basal conditions the lymphatics experience relatively low levels of wall shear stresses, less than 0.5 dynes $/ \mathrm{cm}^{2}$ on average. However, almost immediately upon administration of the fast saline infusion, the average shear stress significantly increases and maximum shear stress rises to $20 \mathrm{dynes} / \mathrm{cm}^{2}$. As speculated earlier, we believe that this increase in shear stress elicits the shear-dependent production of NO, which transitions the vessel from an actively pumping unit to a conduit for fluid transport $[6,7,23,28,46]$. Recently using a similar edemagenic lymphatic in situ model (Bohlen 2011) we have documented that a similar edemagenic hypotonic volume infusion results in increased production of $\mathrm{NO}$ in these lymphatics, which modulates their contractile and pumping function. Given that vessel diameter does not significantly change over time, vessel caliber must be regulated via changes in shear and/or pressure. This supports the idea that lymph vessels appear to have great sensitivities to changes in their mechanical environment (i.e. shear stress, pressure and flow rate) and tend to maintain diameters in situ within a reasonably controlled range, while modulating contraction frequency and pumping efficiency to handle increased fluid volumes immediately after inducing edemagenic stress. A number of ex vivo studies have shown that lymphatic contractility is affected by pressure and/or shear $[6,7,10,11,14,16,18-$

$20,30,31,33,52]$. We believe that this study further highlights the importance of pressure and shear-regulated mechanisms in lymphatic pumping in vivo. The lack of significant increases in vessel diameter is likely due to the highly non-linear character of their pressure-diameter and pressure-volume curves $[11,33,48]$. These vessels are typically highly compliant up to a pressure of approximately $5 \mathrm{cmH}_{2} \mathrm{O}$, where they reach a maximum diameter that remains constant even at much higher pressures as illustrated by Davis et al. [11]. Presumably, this is to limit the amount of lymph that is stored in these conduit vessels [29]. Furthermore, a tenminute saline infusion elicited a major increase in the shear environment up to 30 minutes post infusion. Extrapolating to a larger scale or clinical realms of edema, a sustained elevated level of fluid at the interstitium could take on the order of hours or days to be transported and removed but further investigation is necessary to assess long-term effects of larger edema insults.

Differences between the modest diameter responses observed in this study versus those we have previously seen by Benoit et al. may also be due to the apparent selection of larger lymphatics in this study (average diastolic diameter $\sim 117 \mu \mathrm{m}$ ) compared to those published by Benoit et al. (average diastolic diameter $\sim 77 \mu \mathrm{m}$ ) [4,5]. Previous work by Benoit has also shown that the larger rat mesenteric lymphatics were less sensitive to the effects of hemodilution (i.e. increased fluid volumes) than the smaller mesenteric lymphatics $[4,5]$.

One limitation of this experimental setup is that we did not measure pressure during our experiments due to the complexity of the animal setup. Likewise, we did not want to make it more invasive as that would put the animal at a greater risk of inflammation. Despite this, we believe that pressure did increase at the interstitial tissue space and this is further supported by the work published by Benoit et al. and Cox et al. [4,5,10]. Additionally, we were unable to view and quantify the distance between the initial lymphatics and the location of the imaged lymphatic vessel. It is possible that there are regional variations in the lymphatic response to edemagenic stress and this can be investigated in future studies. 
Another limitation is that in calculating wall shear stress, we assumed Poiseuille flow for simplicity. However we have recently shown that the uncertainty associated with the Poiseuille assumption is $<4 \%$ [32]. Lastly, the frequency presented in this paper is based on the number of contractions observed during acquisition divided by time, providing an "average" value for frequency.

In conclusion, we were able to show that healthy lymphatic vessels in the rat mesentery could sense changes in fluid volume and quickly accommodated to these conditions to transport the excess fluid. We observed significant increases in the lymph pump contraction frequency, lymph flow rate, and average lymphatic wall shear stress, while diameter changes remained relatively modest. These results are highly suggestive that lymphatic vessels are extremely sensitive to mechanical stimuli, which allows them to regulate their ability to adapt to altered fluid conditions.

\section{Acknowledgments}

We would like to acknowledge NIH Grant Nos. R01 HL094269, HL08569 and HL070308 for funding this project and the NSF graduate fellowship for funding E. Rahbar. Additionally, we would like to acknowledge Dr. Mohammad H. Rahbar from the University of Texas Health Science Center at Houston for his guidance and recommendations for statistical analysis.

\section{References}

1. Akl, T.; Rahbar, E.; Zawieja, D.; Gashev, A.; Moore, JE.; Cote, GL. SPIE Vol. 75720K-75720K-6. 2010. Fast imaging system and algorithm for monitoring microlymphatics.

2. Akl TJ, Nagai T, Cote GL, Gashev AA. Mesenteric lymph flow in adult and aged rats. American journal of physiology. Heart and circulatory physiology. 2011; 301:H1828-H1840. [PubMed: 21873496]

3. Akl TJ, Nepiyushchikh ZV, Gashev AA, Zawieja DC, Cot GL. Measuring contraction propagation and localizing pacemaker cells using high speed video microscopy. Journal of biomedical optics. 2011; 16:026016. [PubMed: 21361700]

4. Benoit JN. Relationships between lymphatic pump flow and total lymph flow in the small intestine. The American journal of physiology. 1991; 261:H1970-H1978. [PubMed: 1750545]

5. Benoit JN, Zawieja DC, Goodman AH, Granger HJ. Characterization of intact mesenteric lymphatic pump and its responsiveness to acute edemagenic stress. The American journal of physiology. 1989; 257:H2059-H2069. [PubMed: 2603989]

6. Bohlen HG, Gasheva OY, Zawieja DC. Nitric oxide formation by lymphatic bulb and valves is a major regulatory component of lymphatic pumping. American journal of physiology. Heart and circulatory physiology. 2011; 301:H1897-H1906. [PubMed: 21890688]

7. Bohlen HG, Wang W, Gashev A, Gasheva O, Zawieja D. Phasic contractions of rat mesenteric lymphatics increase basal and phasic nitric oxide generation in vivo. American journal of physiology. Heart and circulatory physiology. 2009; 297:H1319-H1328. [PubMed: 19666850]

8. Chakraborty S, Gurusamy M, Zawieja DC, Muthuchamy M. Lymphatic filariasis: Perspectives on lymphatic remodeling and contractile dysfunction in filarial disease pathogenesis. Microcirculation. 2012

9. Chakraborty S, Zawieja S, Wang W, Zawieja DC, Muthuchamy M. Lymphatic system: a vital link between metabolic syndrome and inflammation. Annals of the New York Academy of Sciences. 2010; 1207(Suppl 1):E94-E102. [PubMed: 20961312]

10. Cox CS Jr, Radhakrishnan R, Villarrubia L, Xue H, Uray K, Gill BS, Stewart RH, Laine GA. Hypertonic saline modulation of intestinal tissue stress and fluid balance. Shock. 2008; 29:598602. [PubMed: 18414233] 
11. Davis MJ, Rahbar E, Gashev AA, Zawieja DC, Moore JE Jr. Determinants of valve gating in collecting lymphatic vessels from rat mesentery. American journal of physiology. Heart and circulatory physiology. 2011; 301:H48-H60. [PubMed: 21460194]

12. DeSantis C, Howlader N, Cronin KA, Jemal A. Breast cancer incidence rates in U.S. women are no longer declining. Cancer epidemiology, biomarkers \& prevention : a publication of the American Association for Cancer Research, cosponsored by the American Society of Preventive Oncology. 2011; 20:733-739.

13. Dixon JB, Gashev AA, Zawieja DC, Moore JE Jr, Cote GL. Image correlation algorithm for measuring lymphocyte velocity and diameter changes in contracting microlymphatics. Annals of biomedical engineering. 2007; 35:387-396. [PubMed: 17151922]

14. Dixon JB, Greiner ST, Gashev AA, Cote GL, Moore JE, Zawieja DC. Lymph flow, shear stress, and lymphocyte velocity in rat mesenteric prenodal lymphatics. Microcirculation. 2006; 13:597610. [PubMed: 16990218]

15. Gashev AA. Physiologic aspects of lymphatic contractile function: current perspectives. Annals of the New York Academy of Sciences. 2002; 979:178-187. discussion 188-196. [PubMed: 12543727]

16. Gashev AA. Lymphatic vessels: pressure- and flow-dependent regulatory reactions. Annals of the New York Academy of Sciences. 2008; 1131:100-109. [PubMed: 18519963]

17. Gashev AA, Davis MJ, Delp MD, Zawieja DC. Regional variations of contractile activity in isolated rat lymphatics. Microcirculation. 2004; 11:477-492. [PubMed: 15371129]

18. Gashev AA, Davis MJ, Zawieja DC. Inhibition of the active lymph pump by flow in rat mesenteric lymphatics and thoracic duct. J Physiol. 2002; 540:1023-1037. [PubMed: 11986387]

19. Gashev AA, Wang W, Laine GA, Stewart RH, Zawieja DC. Characteristics of the active lymph pump in bovine prenodal mesenteric lymphatics. Lymphatic research and biology. 2007; 5:71-79. [PubMed: 17935475]

20. Gashev AA, Zawieja DC. Hydrodynamic regulation of lymphatic transport and the impact of aging. Pathophysiology : the official journal of the International Society for Pathophysiology / ISP. 2010; 17:277-287. [PubMed: 20226639]

21. Gasheva OY, Zawieja DC, Gashev AA. Contraction-initiated NO-dependent lymphatic relaxation: a self-regulatory mechanism in rat thoracic duct. J Physiol. 2006; 575:821-832. [PubMed: 16809357]

22. Ikomi F, Hunt J, Hanna G, Schmid-Schonbein GW. Interstitial fluid, plasma protein, colloid, and leukocyte uptake into initial lymphatics. Journal of applied physiology. 1996; 81:2060-2067. [PubMed: 8941530]

23. Kawai Y, Yokoyama Y, Kaidoh M, Ohhashi T. Shear stress-induced ATP-mediated endothelial constitutive nitric oxide synthase expression in human lymphatic endothelial cells. American journal of physiology. Cell physiology. 2010; 298:C647-C655. [PubMed: 20042732]

24. Levick, JR. An Introduction to Cardiovascular Physiology. London: Hodder Arnold; 2003. Circulation of fluid between plasma, interstitium and lymph.

25. Levick JR. Revision of the Starling principle: new views of tissue fluid balance. J Physiol. 2004; 557:704. [PubMed: 15131237]

26. Levick JR, Michel CC. Microvascular fluid exchange and the revised Starling principle. Cardiovascular research. 2010; 87:198-210. [PubMed: 20200043]

27. Lynch PM, Delano FA, Schmid-Schonbein GW. The primary valves in the initial lymphatics during inflammation. Lymphatic research and biology. 2007; 5:3-10. [PubMed: 17508898]

28. Ohhashi T, Yokoyama S. Nitric oxide and the lymphatic system. The Japanese journal of physiology. 1994; 44:327-342. [PubMed: 7869598]

29. Quick CM, Venugopal AM, Gashev AA, Zawieja DC, Stewart RH. Intrinsic pump-conduit behavior of lymphangions. American journal of physiology. Regulatory, integrative and comparative physiology. 2007; 292:R1510-R1518.

30. Radhakrishnan RS, Radhakrishnan HR, Xue H, Moore-Olufemi SD, Mathur AB, Weisbrodt NW, Moore FA, Allen SJ, Laine GA, Cox CS Jr. Hypertonic saline reverses stiffness in a SpragueDawley rat model of acute intestinal edema, leading to improved intestinal function. Critical care medicine. 2007; 35:538-543. [PubMed: 17205008] 
31. Radhakrishnan RS, Xue H, Moore-Olufemi SD, Weisbrodt NW, Moore FA, Allen SJ, Laine GA, Cox CS Jr. Hypertonic saline resuscitation prevents hydrostatically induced intestinal edema and ileus. Critical care medicine. 2006; 34:1713-1718. [PubMed: 16625118]

32. Rahbar E, Moore JE Jr. A model of a radially expanding and contracting lymphangion. Journal of biomechanics. 2011; 44:1001-1007. [PubMed: 21377158]

33. Rahbar E, Weimer J, Gibbs H, Yeh AT, Bertram CD, Davis MJ, Hill MA, Zawieja DC, Moore JE Jr. Passive pressure-diameter relationship and structural composition of rat mesenteric lymphangions. Lymphatic research and biology. 2012; 10:152-163. [PubMed: 23145980]

34. Rockson SG. Addressing the unmet needs in lymphedema risk management. Lymphatic research and biology. 2006; 4:42-46. [PubMed: 16569207]

35. Rockson SG. Lymphatic research: past, present, and future. Lymphatic research and biology. 2009; 7:183-187. [PubMed: 20143916]

36. Rockson SG. The unique biology of lymphatic edema. Lymphatic research and biology. 2009; 7:97-100. [PubMed: 19522679]

37. Rockson SG. Causes and consequences of lymphatic disease. Annals of the New York Academy of Sciences. 2010; 1207(Suppl 1):E2-E6. [PubMed: 20961302]

38. Rockson SG. The lymphatics and the inflammatory response: lessons learned from human lymphedema. Lymphatic research and biology. 2013; 11:117-120. [PubMed: 24024576]

39. Rockson SG. Lymphatics: where the circulation meets the immune system. Lymphatic research and biology. 2013; 11:115. [PubMed: 24024578]

40. Rockson SG, Rivera KK. Estimating the population burden of lymphedema. Annals of the New York Academy of Sciences. 2008; 1131:147-154. [PubMed: 18519968]

41. Schmid-Schonbein GW. Mechanisms causing initial lymphatics to expand and compress to promote lymph flow. Archives of histology and cytology. 1990; 53(Suppl):107-114. [PubMed: 2252623]

42. Stanton AW, Modi S, Mellor RH, Levick JR, Mortimer PS. Recent advances in breast cancerrelated lymphedema of the arm: lymphatic pump failure and predisposing factors. Lymphatic research and biology. 2009; 7:29-45. [PubMed: 19302022]

43. Szuba A, Rockson SG. Lymphedema: anatomy, physiology and pathogenesis. Vascular medicine. 1997; 2:321-326. [PubMed: 9575606]

44. Szuba A, Rockson SG. Lymphedema: classification, diagnosis and therapy. Vascular medicine. 1998; 3:145-156. [PubMed: 9796078]

45. Trzewik J, Mallipattu SK, Artmann GM, Delano FA, Schmid-Schonbein GW. Evidence for a second valve system in lymphatics: endothelial microvalves. FASEB journal : official publication of the Federation of American Societies for Experimental Biology. 2001; 15:1711-1717. [PubMed: 11481218]

46. Tsunemoto H, Ikomi F, Ohhashi T. Flow-mediated release of nitric oxide from lymphatic endothelial cells of pressurized canine thoracic duct. The Japanese journal of physiology. 2003; 53:157-163. [PubMed: 14529576]

47. Venugopal AM, Stewart RH, Laine GA, Dongaonkar RM, Quick CM. Lymphangion coordination minimally affects mean flow in lymphatic vessels. American journal of physiology. Heart and circulatory physiology. 2007; 293:H1183-H1189. [PubMed: 17468331]

48. Venugopal AM, Stewart RH, Laine GA, Quick CM. Nonlinear lymphangion pressurevolume relationship minimizes edema. American journal of physiology. Heart and circulatory physiology. 2010; 299:H876-H882. [PubMed: 20601461]

49. World Heath Organization W. Report on the mid-term assessment of microfilaraemia reduction in sentinel sites of 13 countries of the Global Programme to Eliminate Lymphatic Filariasis. 2004.

50. Zawieja, D.; von der Weid, P.; Gashev, A. Microlymphatic Biology. In: Terjung, R., editor. Comprehensive Physiology. Wiley; 2011. p. 125-158.

51. Zawieja DC. Lymphatic microcirculation. Microcirculation. 1996; 3:241-243. [PubMed: 8839448]

52. Zawieja DC. Contractile physiology of lymphatics. Lymphatic research and biology. 2009; 7:8796. [PubMed: 19534632] 
53. Zawieja DC, Davis KL, Schuster R, Hinds WM, Granger HJ. Distribution, propagation, and coordination of contractile activity in lymphatics. The American journal of physiology. 1993; 264:H1283-H1291. [PubMed: 8476104] 

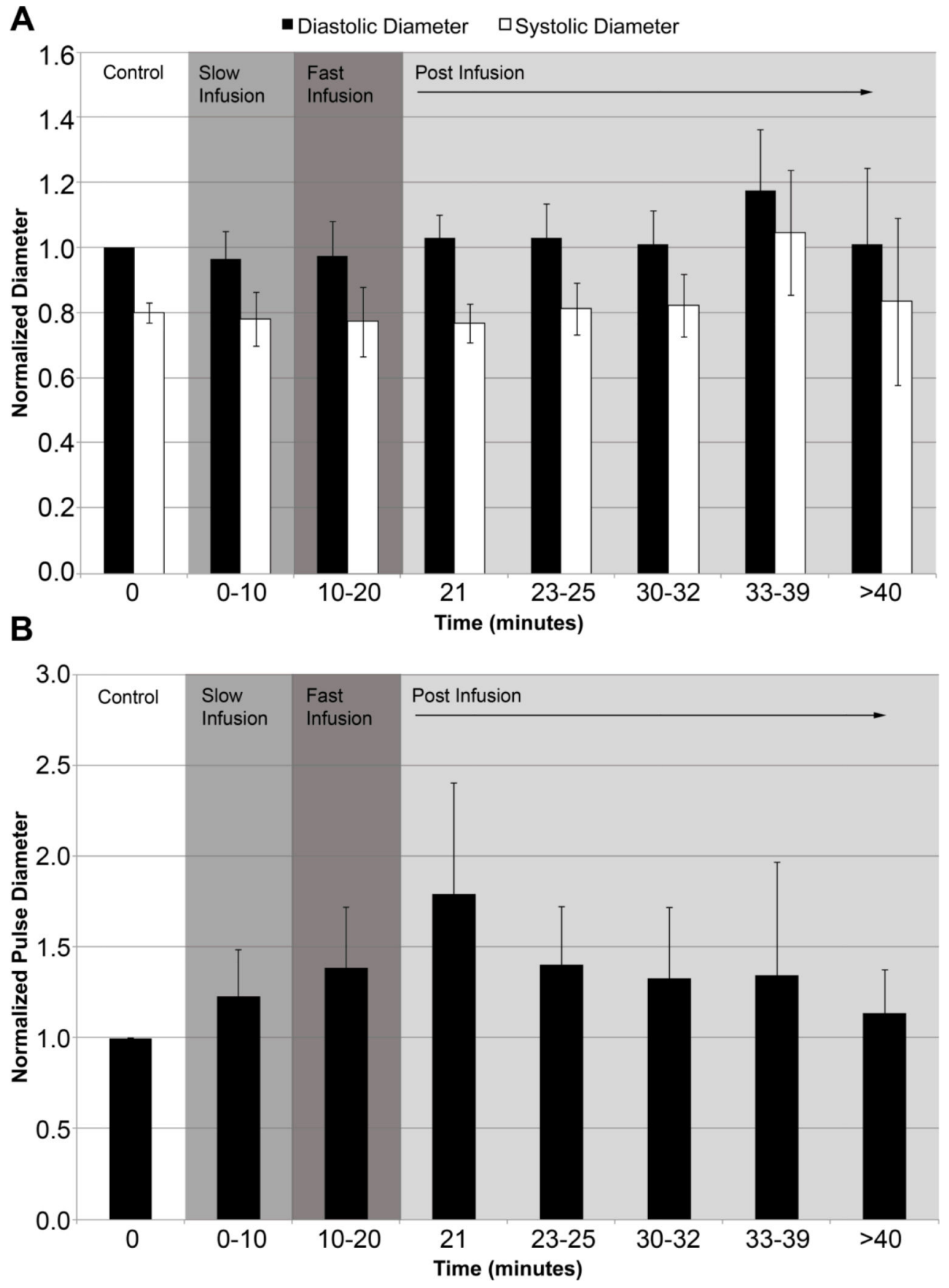

Figure 1.

Plots of normalized Dd, Ds and pulse diameter. (A) Diastolic (black) and systolic (white) diameters were normalized to the control diastolic diameter. (B) Normalized pulse diameter over the entire duration of the experiment. Changes in diameter were not statistically significant ( $\mathrm{p}$-value $>0.05$ ). 
- Average Velocity (mm/s) $\quad \square$ Average Flow Rate (uL/min)

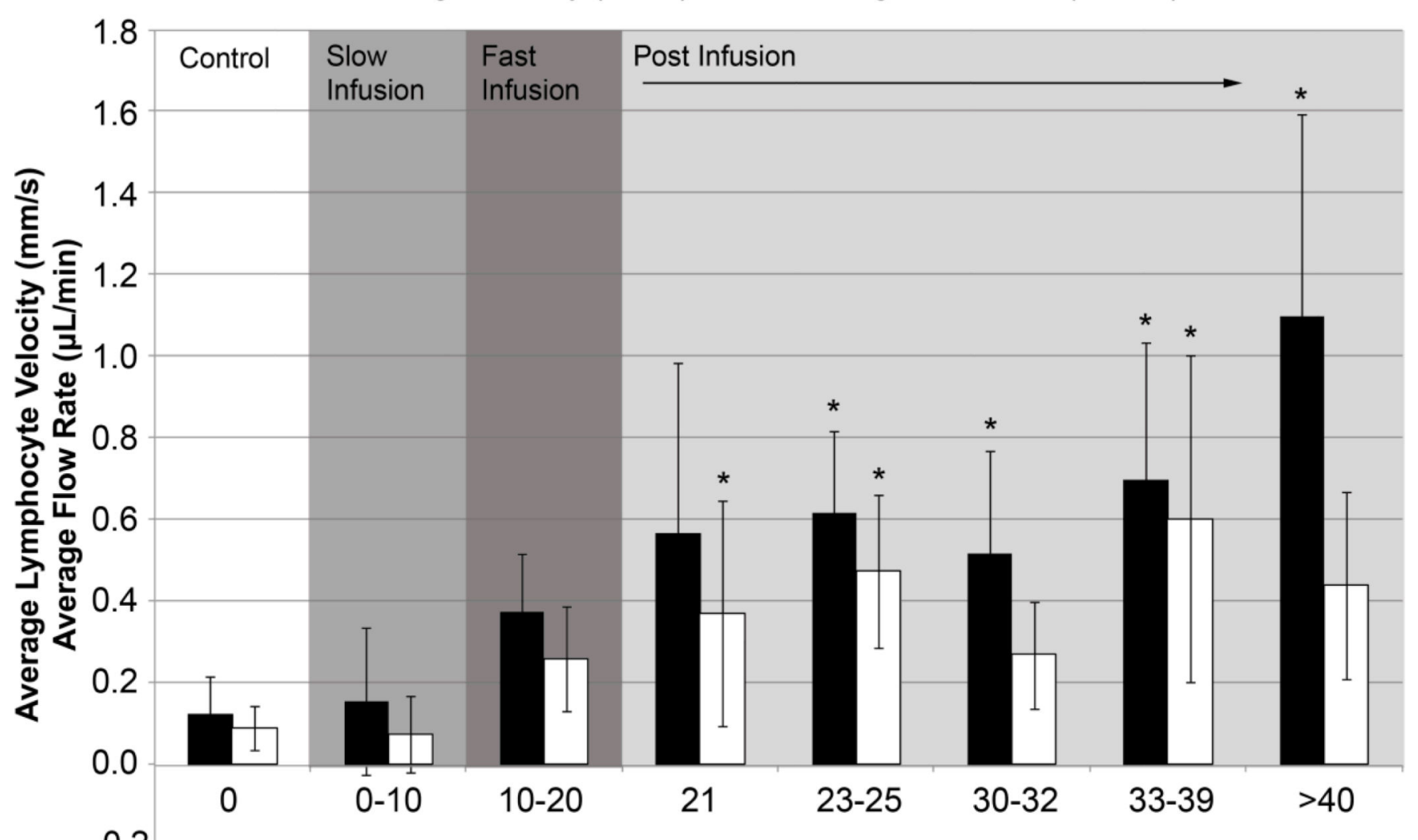

$-0.2$

\section{Time (minutes)}

Figure 2.

Plots of average lymphocyte velocity and flow rate. Average lymphocyte velocity and flow rate were measured throughout the experiment up to 50 minutes from the start of the experiment. Asterisks indicate a statistical increase compared to control values at time $=0$ ( $p$ value< 0.05$)$. 


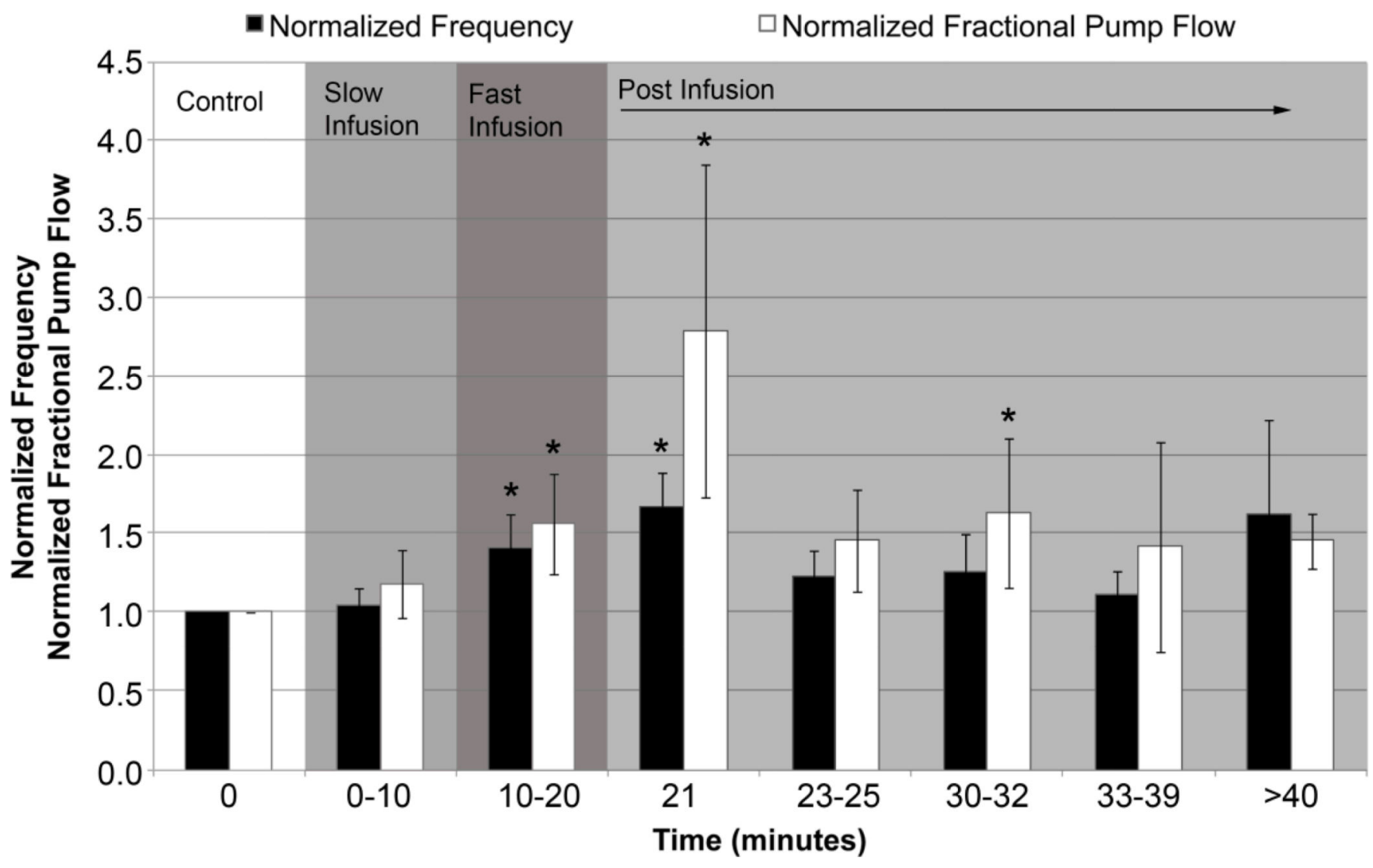

Figure 3.

Plots of normalized contraction frequency and fractional pump flow. Contraction frequency and fractional pump flow were normalized to control values. Asterisks indicate a statistically significant increase in each parameter with respect to the control value at time $=0$ ( $\mathrm{p}$-value $<$ $0.05)$. 


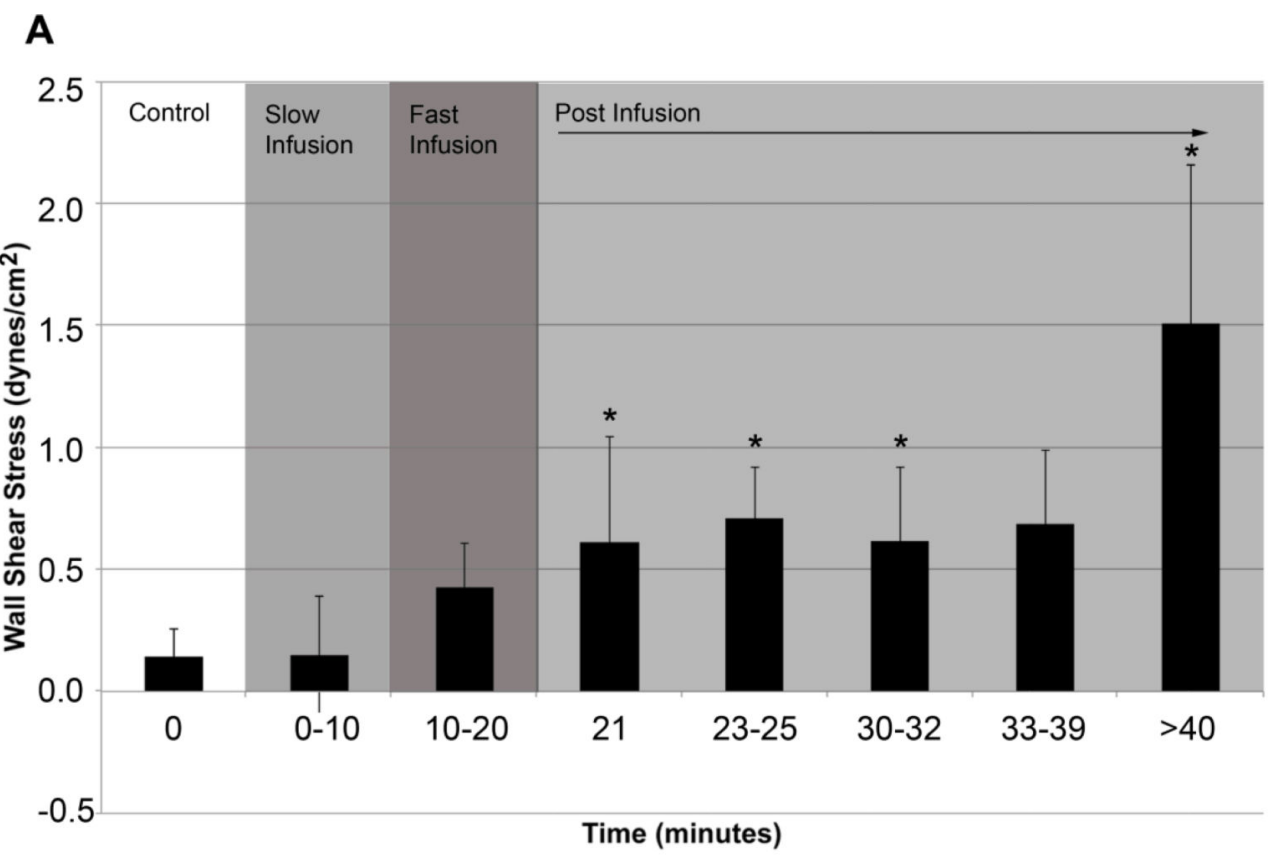

\section{B}

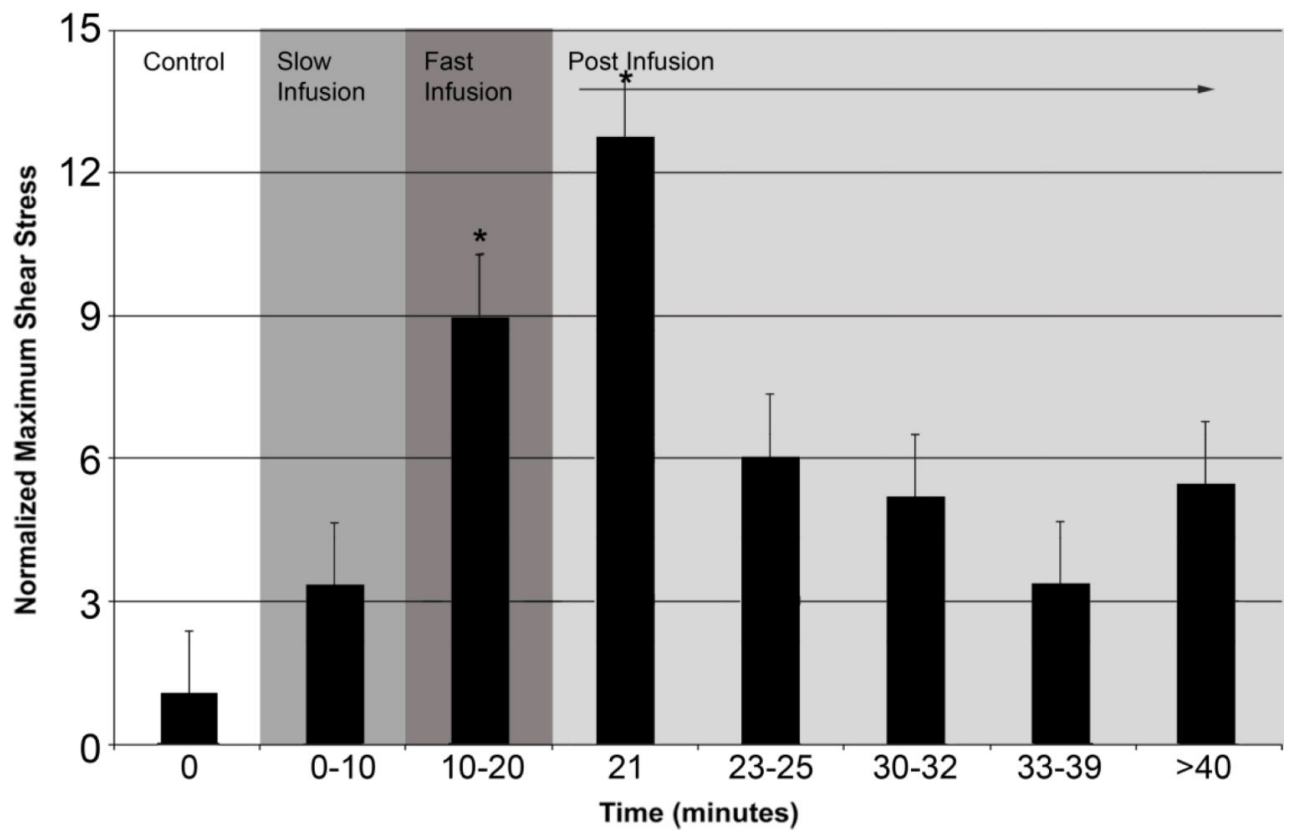

Figure 4.

Plots of average wall shear stress and normalized maximum wall shear stress. (A) Average wall shear stress calculated assuming Poiseuille flow. (B) Maximum wall shear stress normalized to control. Asterisks indicate a statistically significant increase in shear stress (pvalue $<0.05)$. Note that shear stress was immediately affected with the induction of the fast infusion. 


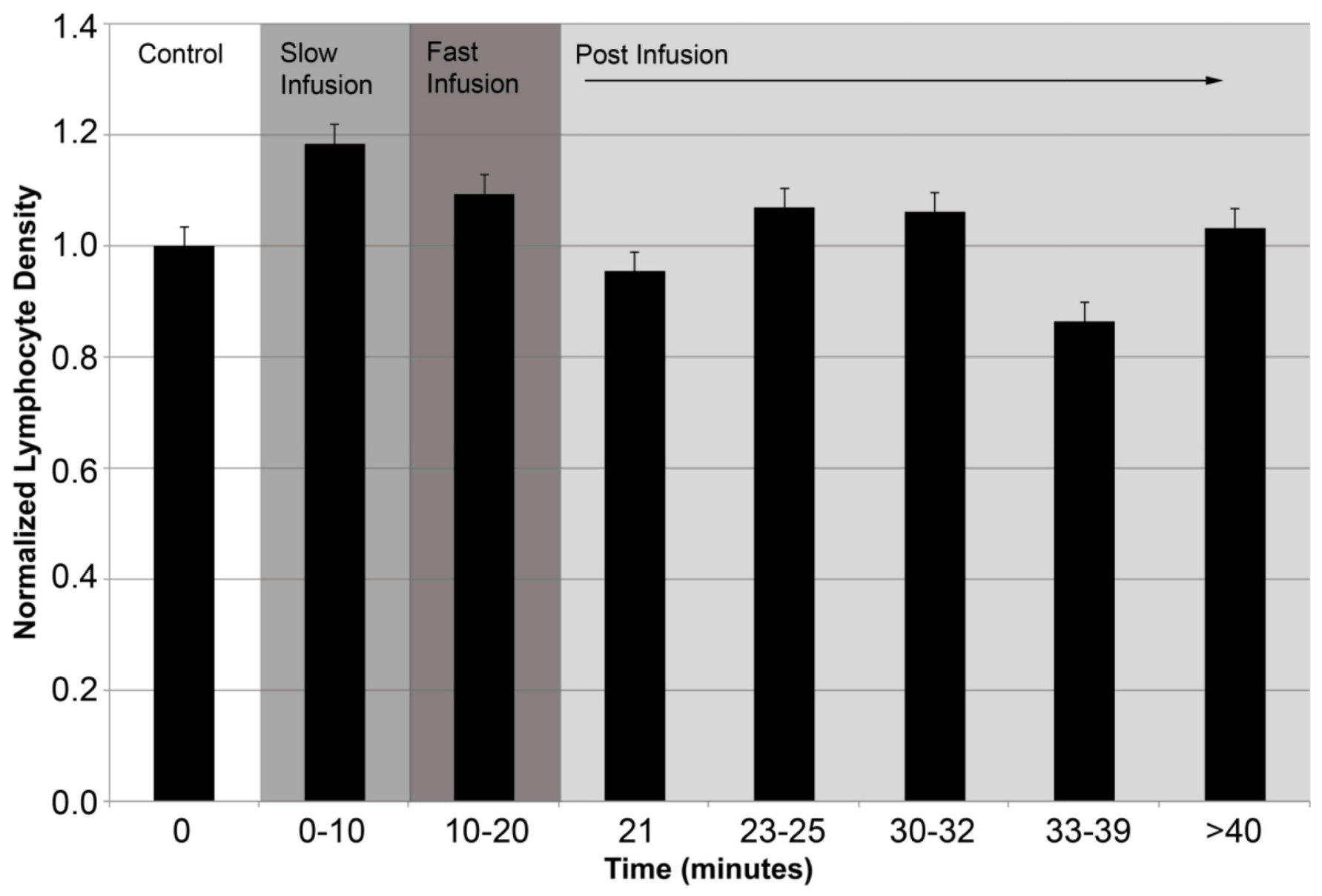

Figure 5.

Plots of normalized lymphocyte density. No statistically significant change in density was observed (p-value $>0.05$ ). However, a $15 \%$ reduction in cells was observed post-infusion (33-39 minutes) as was expected by the dilution of lymph via the saline infusion. 